\title{
Anharmonicity Effects in Nanocrystals Studied by Raman Scattering Spectroscopy
}

\author{
Z. Dohčević-Mitrović* ${ }^{*}$ Z.V. Popović And M. Šćepanović \\ Center for Solid State Physics and New Materials, Institute of Physics, Belgrade, Serbia
}

\begin{abstract}
Phonon-phonon interactions were investigated in various nanocrystalline powders like anatase $\mathrm{TiO}_{2-\delta}$, pure $\mathrm{CeO}_{2-\delta}$ and ceria doped with $\mathrm{Nd}(\mathrm{Gd})$ analyzing temperature dependent Raman spectra of these systems. Phonon confinement model based on size, inhomogeneous strain and anharmonic effects was used to properly describe the evident changes present in the Raman spectra of pure and doped ceria nanocrystalline samples. In small particles of pure and doped ceria nanocrystals, when size effects have minor impact on Raman modes, four phonon anharmonic processes prevail under the three-phonon ones. When nanopowdered particles are grown enough size effects provoke changes of the anharmonic interactions when three-phonon coupling prevails over the four-phonon anharmonic processes. In nanocrystalline anatase $\mathrm{TiO}_{2}$ evident blueshift of the most prominent $E_{\mathrm{g}}$ Raman mode probably originates from dominant four-phonon anharmonic interactions.
\end{abstract}

PACS numbers: 63.22.-m, 78.67.Bf, 78.30.-j, 61.72.uj

\section{Introduction}

Physical properties of materials are dependent on temperature. Temperature affects the population of the different levels for each normal mode provoking the change of the lattice parameters. Any change of the lattice parameters with temperature is attributed to the anharmonicity of the lattice potential. As an effect of the anharmonic interaction, a nonequilibrium phonon population decays into phonons of lower energy or is scattered by thermal phonons into modes of different frequencies.

The Raman spectra of various amorphous or crystalline materials show changes in line position and bandwidth with temperature [1-4]. This changes manifest in shift of line position and a change in line width and intensity. Any frequency shift or the variation of the half-width $\Gamma$ with temperature of the Raman modes are the measurements of the anharmonicity of the interactions. The phonon-phonon interactions are of special interest in nanostructured systems if we have in mind that in the literature there is still no adequate theory which describes the anharmonic effects in these systems $[5,6]$.

We investigated the anharmonic behaviour of the first-order Raman modes in vaious nanocrystalline materials like anatase $\mathrm{TiO}_{2-\delta}$, pure $\mathrm{CeO}_{2-\delta}$, and ceria doped with rare earth elements like Nd and Gd by the Raman spectroscopy method. The aim of this work was to examine the temperature-induced changes of the vibrational properties of these materials and to better understand the contribution of volume expansion and anharmonic intermode coupling to the Raman frequency shift and line width in these systems.

* corresponding author; e-mail: zordoh@phy.bg.ac.yu

\section{Experiment}

Pure ceria nanocrystalline sample as well as ceria samples doped with $\mathrm{Nd}(\mathrm{Gd})$ were synthesized by self-propagating room temperature synthesis. The detailed powder preparation procedure is already described [7]. Sample of nanocrystalline anatase $\mathrm{TiO}_{2}$ was supplied by Merck.

The vibrational properties of these nanopowders were studied by the Raman spectroscopy method. Micro-Raman spectra were collected in backscattering configuration using Jobin Yvon T64000 spectrometer equipped with a nitrogen cooled charge-coupled-device detector. Argon ion $\left(\mathrm{Ar}^{+}\right)$laser line $\lambda=514.5 \mathrm{~nm}$ was used as an excitation source. The pure and doped ceria samples and $\mathrm{TiO}_{2}$ sample were progressively heated in Linkam TS 1500 microscope heating stage, up to $1073 \mathrm{~K}$ and then gradually cooled down to room temperature.

\section{Results and discussion}

The variation of the frequency of the normal modes with temperature at constant pressure arises from two contributions: pure volume contribution which results from thermal expansion and pure-temperature contribution which results from cubic and quartic anharmonicities (self-energy shift). The complete expression for the frequency as a function of temperature can be expressed as

$$
\begin{aligned}
& \omega(T)=\omega_{0}+\Delta_{\mathrm{E}}(q j)+\Delta_{\mathrm{A}}(q j, \Omega)= \\
& \omega_{0}+\Delta_{\mathrm{E}}+\Delta_{3}+\Delta_{4},
\end{aligned}
$$

where $\omega_{0}$ is harmonic frequency, term $\Delta_{\mathrm{E}}$ arises from the thermal expansion while $\Delta_{\mathrm{A}}$ represents pure temperature contribution due to cubic $\left(\Delta_{3}\right)$ and quartic anharmonicities $\left(\Delta_{4}\right)$. In most solids $\Delta_{\mathrm{E}}<0$ i.e. lattice dilation results in mode softening. Frequency shift due to thermal 
expansion can be evaluated from the following equation:

$$
\Delta_{\mathrm{E}}=\omega_{0}\left[\exp \left(-3 \gamma_{i} \int_{0}^{T} \alpha\left(T^{\prime}\right) \mathrm{d} T^{\prime}\right)-1\right] .
$$

Frequency shifts $\Delta_{3}$ and $\Delta_{4}$ arise from phonon-phonon interactions due to the lowest-order cubic and quartic terms in the interatomic potential. The multiphonon processes associated with the cubic terms give rise to a negative frequency shift $\Delta_{3}<0$, whereas the shift $\Delta_{4}$ associated with quartic anharmonicity can be either positive or negative [8]. The resultant frequency shift due to phonon-phonon interaction may be either positive or negative depending on the relative magnitudes of the anharmonic terms in the interatomic potential. The expression for $\Delta_{\mathrm{A}}$ arising from the cubic and quartic terms in the first approximation, is given [8]:

$$
\begin{aligned}
\Delta_{\mathrm{A}} & =-\frac{18}{\hbar^{2}} \sum_{q_{1} q_{2} j_{1} j_{2}}\left|V\left(\begin{array}{ccc}
0 & q_{1} & q_{2} \\
j & J_{1} & j_{2}
\end{array}\right)\right|^{2}\left(\frac{n_{1}+n_{2}+1}{\omega_{1}+\omega_{2}+1}\right. \\
& \left.+\frac{n_{1}+n_{2}+1}{\omega_{1}+\omega_{2}-\Omega}+\frac{n_{2}-n_{1}}{\omega_{1}-\omega_{2}+\Omega}+\frac{n_{2}-1}{\omega_{1}-\omega_{2}-\Omega}\right) \\
& +\frac{12}{\hbar^{2}} \sum_{q_{1} j_{1}} V\left(\begin{array}{cccc}
0 & 0 & q_{1} & -q_{1} \\
j & j & j_{1} & j_{1}
\end{array}\right)\left(2 n_{1}+1\right) .
\end{aligned}
$$

The first term in Eq. (3) represents the cubic anharmonic term which comprises down and up conversion processes [9] whereas the second term relates to the first-order quartic term which is always positive. The $n_{j}(q)$ is the Bose-Einstein occupation factor of the $j$-th phonon mode with wave vector $q$ and frequency $\omega$ given as

$$
n(T, \omega)=\exp \left(\frac{\hbar \omega}{k_{\mathrm{B}} T}\right)-1 .
$$

Klemens assumed that the optical Raman phonon decays in two acoustical phonons of opposite $q$ belonging to the same branch $\left(j_{1}=j_{2}\right)$. In this case the equations for frequency and bandwidth change of Raman modes with temperature including three phonon-processes are simplified [10]:

$$
\begin{aligned}
& \Delta(T)=A\left(1+\frac{2}{\mathrm{e}^{\hbar \omega / 2 k_{\mathrm{B}} T}-1}\right), \\
& \Delta \Gamma(T)=C\left(1+\frac{2}{\mathrm{e}^{\hbar \omega / 2 k_{\mathrm{B}} T}-1}\right) .
\end{aligned}
$$

Although this model is a great oversimplification, it has gained wide acceptance, specially after the work of Hart et al. on $\mathrm{Si}$ [11]. Balkanski et al. performed systematic temperature measurements of the line width and frequency shift of the Raman LO phonon in Si for the temperature range $5-1400 \mathrm{~K}$ [12] and deduced that at higher temperatures besides cubic terms the incorporation of quartic anharmonicity terms give satisfactory fits of the experimental values. In this case the frequency and bandwidth change of Raman modes with temperature looks like [12]:

$$
\Delta(T)=A\left(1+\frac{2}{\mathrm{e}^{\hbar \omega / 2 k_{\mathrm{B}} T}-1}\right)
$$

$$
\begin{gathered}
+B\left[1+\frac{3}{\mathrm{e}^{\hbar \omega / 3 k_{\mathrm{B}} T}-1}+\frac{3}{\left(\mathrm{e}^{\hbar \omega / 3 k_{\mathrm{B}} T}-1\right)^{2}}\right], \\
\Delta \Gamma(T)=C\left(1+\frac{2}{\mathrm{e}^{\hbar \omega / 2 k_{\mathrm{B}} T}-1}\right) \\
+D\left[1+\frac{3}{\mathrm{e}^{\hbar \omega / 3 k_{\mathrm{B}} T}-1}+\frac{3}{\left(\mathrm{e}^{\hbar \omega / 3 k_{\mathrm{B}} T}-1\right)^{2}}\right],
\end{gathered}
$$

where $A, B, C, D$ are anharmonic constants.

\subsection{Anharmonic effects in $\mathrm{CeO}_{2-\delta}$ nanocrystals}

In Fig. 1 there are given Raman spectra of $\mathrm{CeO}_{2-\delta}$ nanocrystals measured at different temperatures starting from room temperature (RT) up to $1273 \mathrm{~K}$ [13]. By heating, the $F_{2 \mathrm{~g}}$ Raman mode frequency continuously decreases and its line shape becomes less asymmetric indicating the change in crystallite size.

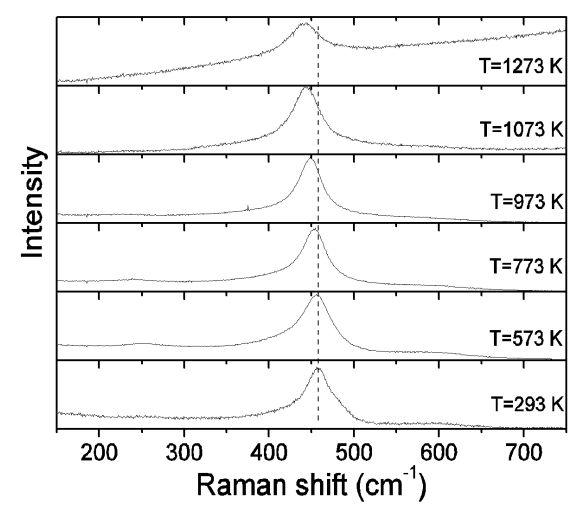

Fig. 1. Raman spectra of $\mathrm{CeO}_{2-\delta}$ nanocrystals at different temperatures.

Namely, the particle size effect provokes systematic changes in the Raman spectra of $\mathrm{CeO}_{2-\delta}$ nanocrystals when crystallite size decreases [14-16]. The evolution of the $F_{2 \mathrm{~g}}$ Raman mode (the mode frequency shift and asymmetrical broadening) strongly depends on the confinement and inhomogeneous strain effects due to the particle size change and is best described using phonon-confinement model (PCM) [17, 18]. On the other side, the temperature provokes the changes of the Raman line position and bandwidth. Such temperature dependence can be attributed to the phonon-phonon interactions originating from crystal anharmonicity. As an effect of anharmonic interactions an optical mode can interchange energy with other lattice modes in such a way that it decays to lower energy phonons or is scattered by thermal phonons into modes of different energy. Figure 2 shows the first-order Raman mode frequency and bandwidth change of first-order $F_{2 \mathrm{~g}}$ Raman mode in $\mathrm{CeO}_{2-\delta}$ nanocrystals at different temperatures.

By heating, the Raman frequency continuously decreases (circles in Fig. 2). In addition, as the temperature increases, the line shape becomes less asymmetric, and at about $1100 \mathrm{~K}$ it becomes totally symmetric, as 


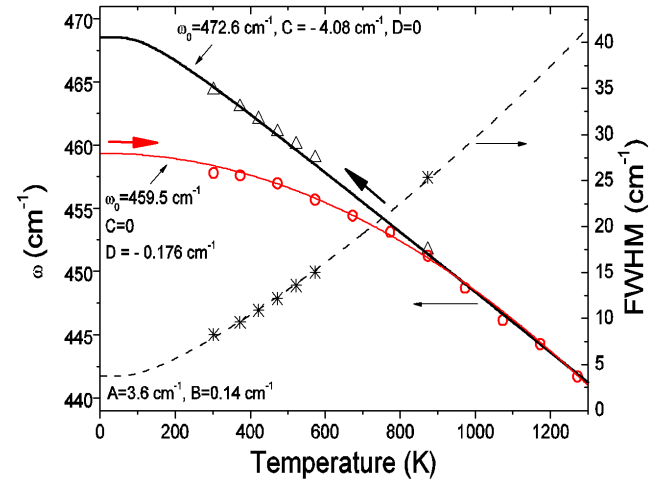

Fig. 2. Frequency (circles, triangles) and line width (asterisks) change of the $F_{2 g}$ Raman mode with temperature. The solid (dashed) lines are calculated data using Eqs. (6) and (7).

can be seen from Fig. 1. By cooling, the frequency of the $F_{2 \mathrm{~g}}$ mode increases (triangles in Fig. 2), reaching the value of $464 \mathrm{~cm}^{-1}$ at room temperature very close to the value of $\mathrm{CeO}_{2}$ single crystals $[19,20]$ while the line width decreases with temperature decrease. Temperature dependence of the Raman mode frequency and bandwidth based on the Klemens ansatz [10], was fitted using Eqs. (6) and (7). The results of the fitting procedure (full line) applied to the experimental frequency dependence on temperature during the heating demonstrated that the anharmonicity is dominated by four-phonon anharmonic processes. Namely, as can be seen from Fig. 2, the Raman mode frequency of the nanocrystals (circles) varies as $T^{2}\left(C=0, D=-0.176 \mathrm{~cm}^{-1}\right)$. We concluded that in $\mathrm{CeO}_{2-\delta}$ nanocrystals, the anharmonicity is dominated by different phonon decay processes (four-phonon processes) [13]. Upon gradual cooling down to room temperature the same fitting analysis applied to frequency (open triangles) and line width (asterix) experimental values showed that three phonon processes became dominant (see fitting parameters in Fig. 2). Such behavior pointed out that our sample suffers a transformation from nano to polycrystalline one by above mentioned heating treatment. The subtle interplay between size and anharmonic effects, depending on the heat treatment, leads to different phonon-phonon interactions in nanostructured systems under consideration.

\subsection{High temperature Raman study of $\mathrm{Ce}_{0.75} \mathrm{Nd}_{0.25} \mathrm{O}_{2-\delta}$ nanocrystals}

The Raman spectra of $\mathrm{Ce}_{0.75} \mathrm{Nd}_{0.25} \mathrm{O}_{2-\delta}$ sample obtained upon heating (circles) and cooling (dashed lines) are presented in Fig. 3. Raman spectra of the $\mathrm{Ce}_{0.75} \mathrm{Nd}_{0.25} \mathrm{O}_{2-\delta}$ sample from Fig. 3 were analyzed using the phonon confinement model-PCM that incorporates size, inhomogeneous strain and anharmonic effects [21].

In the PCM there are included inhomogeneous strain effect through the term $\Delta \omega(q, L)$ using Gaussian particle size distribution function $\rho(L)$ and anharmonic effects

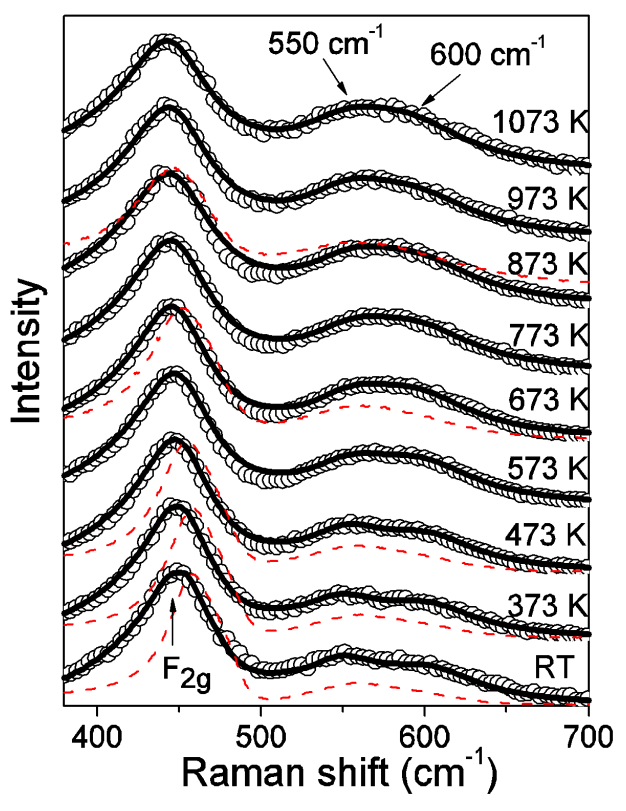

Fig. 3. Raman spectra of $\mathrm{Ce}_{0.75} \mathrm{Nd}_{0.25} \mathrm{O}_{2-\delta}$ sample obtained upon heating (circles) and cooling (dashed lines). The solid lines are calculated spectra using PCM model.

through terms $\Delta \omega(T), \Gamma(T)$ :

$$
\begin{aligned}
& I(\omega)=\sum_{i=1}^{3} \int_{0}^{\infty} \rho(L) \mathrm{d} L \\
& \quad \times \int_{\mathrm{BZ}} \frac{\exp \left(-\frac{q^{2} L^{2}}{8 \beta}\right) \mathrm{d}^{3} q}{\left[\omega-\left(\omega_{i}(q)+\Delta \omega\right)\right]^{2}+[\Gamma(T) / 2]^{2}},
\end{aligned}
$$

where the wave vector $q$ is expressed in units of $2 \pi / a$ ( $a$ is the lattice constant), $L$ is the particle diameter, $\Delta \omega=\Delta \omega_{i}(q, L)+\Delta \omega(T)$ and $\beta$ is an adjustable parameter concerning the strength of the phonon confinement in nanomaterials. The full lines in Fig. 3 are the calculated spectra based on Eq. (8). Using the PCM that incorporates anharmonic and size effects we were able to calculate the change of average particle size at higher temperatures and to estimate the particle growth influence on the $F_{2 \mathrm{~g}}$ Raman mode behaviour. From the PCM model we obtained that particle size increased from $6 \mathrm{~nm}$ to $12 \mathrm{~nm}$ during the heating [21]. The detailed study of the frequency (bandwidth) behavior upon heating revealed that four-phonon anharmonic processes dominate over size effects and three-phonon anharmonic processes in nanocrystalline grains (see Fig. 4a) similar to pure ceria nanocrystals. On the other side, if we take into account that particles grow during the heating we would expect the upshift of the $F_{2 \mathrm{~g}}$ mode frequency (triangles in Fig. 4a) and line width decrease. Experimentally evident redshift and broadening of the $F_{2 \mathrm{~g}}$ mode during heating, clearly points to the fact that anharmonicity effects at higher temperatures influence more the frequency (line width) change than size effects [21]. The temperature dependence of $F_{2 \mathrm{~g}}$ mode frequency and line width upon 

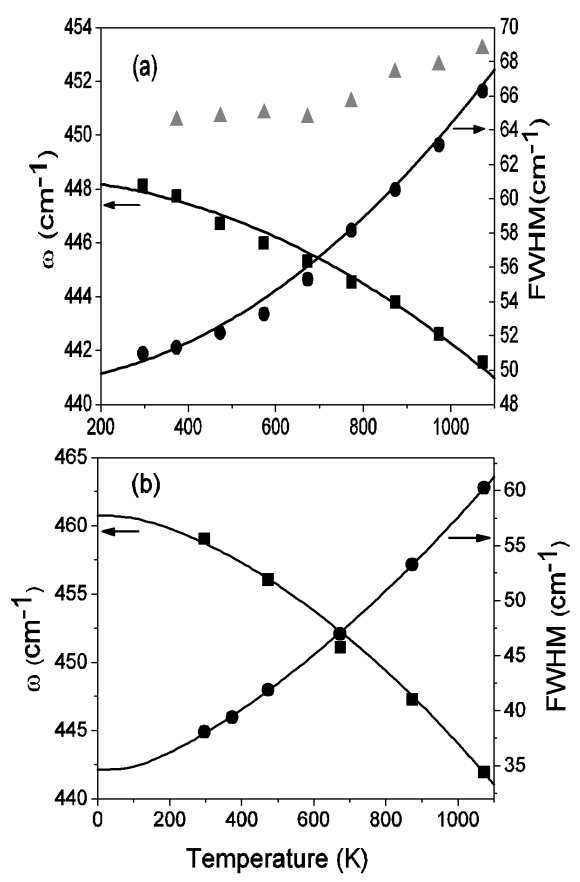

Fig. 4. Frequency and line width change (a) upon heating and (b) upon gradual cooling down to room temperature. The solid lines represent calculated data for the frequency (bandwidth) change with temperature. The best fitting parameters are given in Ref. [21].

cooling (see Fig. 4b) is similar to microceria sample where three phonon processes became dominant [21].

\subsection{Phonon-phonon interactions in $\mathrm{Ce}_{0.85} \mathrm{Gd}_{0.15} \mathrm{O}_{2-\delta}$ nanocrystals}

The Raman spectra (circles) of $\mathrm{Ce}_{0.85} \mathrm{Gd}_{0.15} \mathrm{O}_{2-\delta}$ nanocrystalline sample from Fig. 5 in the temperature range $293-1100 \mathrm{~K}$ exhibit significant changes similar to pure and Nd-doped ceria samples. With increasing temperature the $F_{2 g}$ mode shifts to lower frequencies and becomes less asymmetric while the line width increases. Such a behavior can be attributed to the anharmonicity interactions at elevated temperatures. The Raman spectra (circles) are analyzed by PCM model (Eq. (8)) and the calculated spectra are presented with solid lines. Detailed analysis of frequency (line width) behavior with temperature revealed the change of the phonon-phonon coupling in this system. Namely, careful analysis of the evolution of $F_{2 g}$ Raman mode with temperature showed that temperature dependence of the bandwidth, given in Fig. $6 \mathrm{~b}$, is determined by four-phonon anharmonicity up to the $573 \mathrm{~K}$ (full line fit).

At higher temperatures when the particles are grown enough size effects provoke the changes in phononphonon interactions influencing the bandwidth of the Raman mode in such a way that three-phonon anharmonic interactions prevail over the four-phonon ones (dashed line fit). The best fit of the frequency (full line) during the heating up to $573 \mathrm{~K}$ was obtained including only

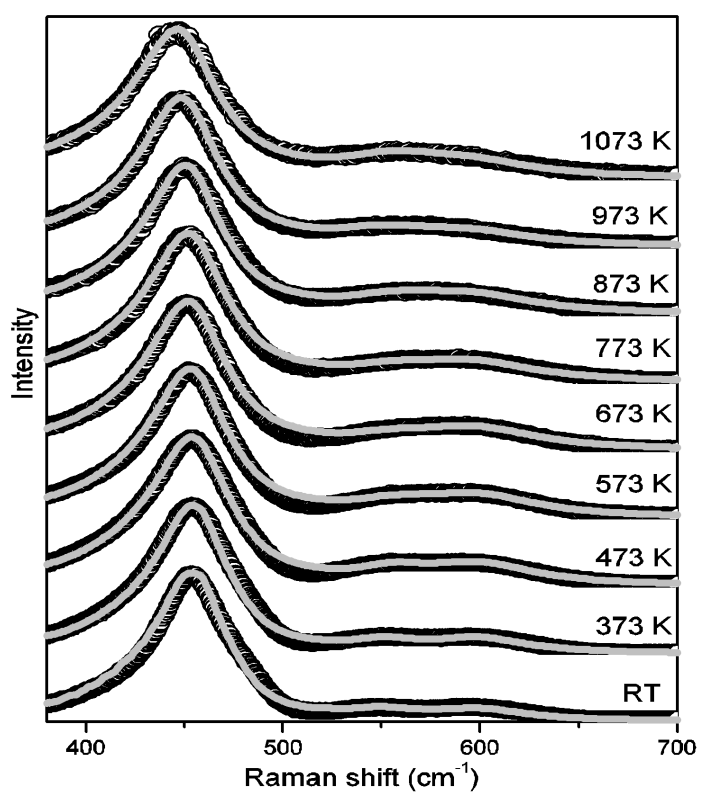

Fig. 5. Raman spectra of $\mathrm{Ce}_{0.85} \mathrm{Gd}_{0.15} \mathrm{O}_{2-\delta}$ sample at different temperatures (circles). The solid lines are calculated spectra using PCM.

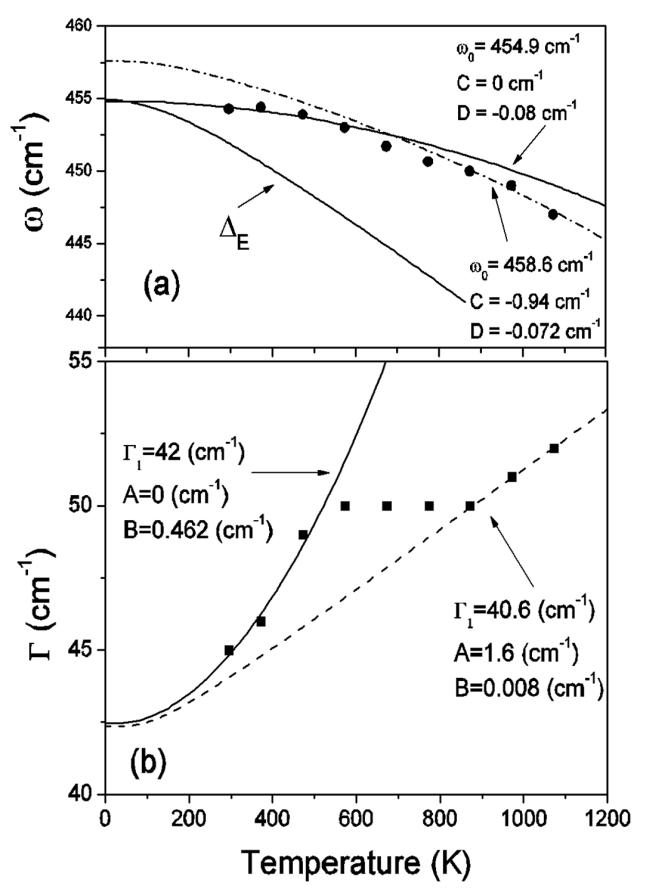

Fig. 6. Temperature dependence of the $F_{2 g}$ Raman mode (a) frequency and (b) line width during heating.

the four-phonon anharmonic term (see Fig. 6a) while at higher temperatures three phonon decay processes became dominant (dashed line). Anharmonic fitting parameters for both quantities are also presented in Fig. 6a and b. From such an analysis, we concluded that different phonon decay channels dominate in nanocrystalline 
grain than in larger grain and pure anharmonicity effects at temperatures up to $573 \mathrm{~K}$ have more influence on the frequency (bandwidth) than size effects [22]. At temperatures higher than $573 \mathrm{~K}$ three phonon processes became dominant. In Fig. 6a there is also presented the calculated frequency shift due to the pure-volume contribution - $\Delta_{\mathrm{E}}$ (line) [23]. The frequency redshift due to the thermal expansion would be higher than the experimentally obtained frequency shift. This suggests that anharmonic couplings of phonons influence more the frequency behaviour of $F_{2 g}$ Raman mode during heating than thermal expansion. The Raman spectrum at room temperature after cooling (Fig. 7a) is narrower, blue shifted regarding the starting spectrum but still at lower frequency than in the bulk counterpart meaning that particles are in nanometric range. Upon cooling frequency shifts to higher values while bandwidth decreases (Fig. 7a). The best fit of the frequency (line width) behaviour with cooling down to room temperature, presented in Fig. 7b with full (dashed) lines, was obtained including three phonon anharmonic processes which become dominant. This implies that the particles are grown enough so the phononphonon interactions are more similar to polycrystalline ceria [22].

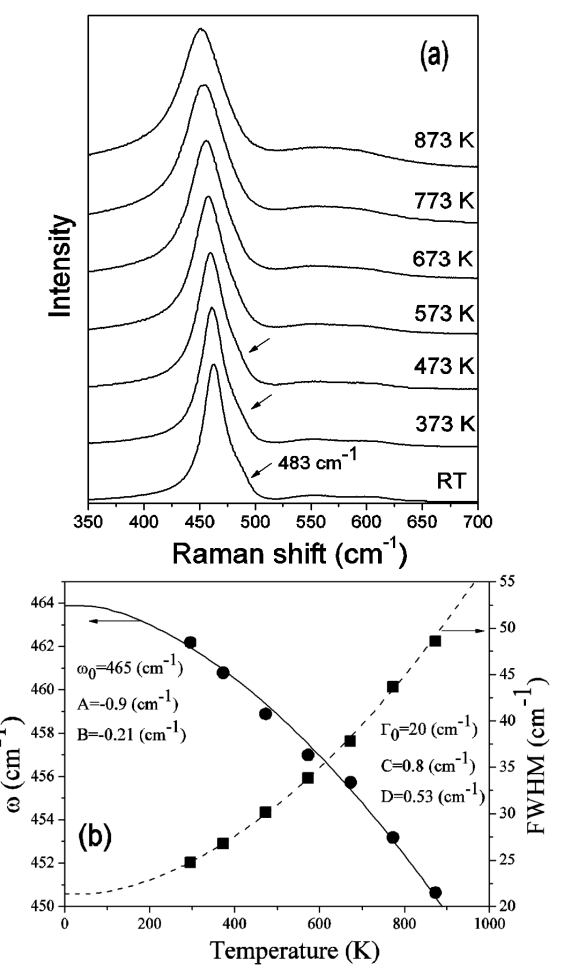

Fig. 7. (a) Raman spectra of $\mathrm{Ce}_{0.85} \mathrm{Gd}_{0.15} \mathrm{O}_{2-\delta}$ sample upon gradual cooling down to room temperature and (b) variation of the $F_{2 \mathrm{~g}}$ mode frequency and line width upon gradual cooling. Full (dashed) lines represent fits of the line position and line width using Eqs. (6) and (7).

\subsection{Intrinsic anharmonicity in nanocrystalline anatase $\mathrm{TiO}_{2}$}

$\mathrm{TiO}_{2}$ in anatase phase has six first-order Raman active modes among which low frequency $E_{\mathrm{g}(1)}$ and $E_{\mathrm{g}(2)}$ modes exhibit frequency shift up (hardening behavior) with increasing temperature while the other four modes exhibit softening behavior with rising temperature [24-26]. In Fig. 8 there is presented the temperature dependence of the $E_{\mathrm{g}(1)}$ mode frequency. The Raman shift dependence on temperature can be attributed to two effects, one being the thermal expansion or volume change and the other optical-phonon anharmonic couplings. The variation of the frequency of the Raman modes with temperature at constant pressure arises from two contributions: pure volume contribution which results from thermal expansion and pure-temperature contribution which results from cubic and quartic anharmonicities. Temperature dependence of each vibrational mode at constant pressure can be expressed

$$
\begin{aligned}
& (\partial \ln \omega / \partial \ln T) p=-(\alpha / k)(\partial \ln \omega / \partial \ln P)_{T} \\
& \quad+(\partial \ln \omega / \partial \ln T)_{V}
\end{aligned}
$$

where $\alpha$ is the volume thermal expansion coefficient and $k$ is the isothermal volume compressibility. Above equation can be written in terms of measured pressure and temperature dependence of the Raman-active mode as

$$
\begin{gathered}
\left(\Delta \omega_{T}\right)_{P}=-\left(\Delta \omega_{P}\right)_{T}+\left(\Delta \omega_{T}\right)_{V}= \\
\Delta_{\mathrm{E}}(q j)+\Delta_{\mathrm{A}}(q j, \Omega) .
\end{gathered}
$$

In his work Osaka measured pressure and temperature dependence of each anatase mode and was able to separate the thermal expansion contribution from pure phonon-phonon interactions and to estimate their influence on each Raman mode in anatase $\mathrm{TiO}_{2}$ [25]. From this study he deduced, as well as later Gao in nanocrystalline anatase [26], that anharmonic couplings of optical phonons become much stronger upon increasing temperature in the $E_{\mathrm{g}(1)}$ mode than quasiharmonic contribution arising from thermal expansion. Both authors deduced that strongly intrinsic anharmonicity is responsible for

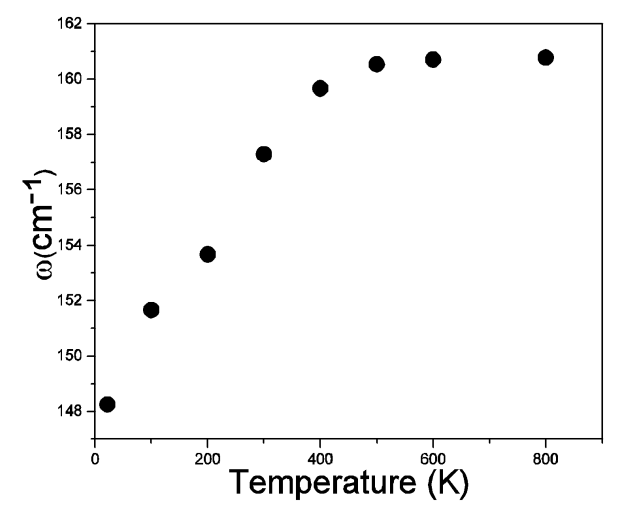

Fig. 8. Frequency of the $E_{\mathrm{g}(1)}$ mode versus temperature. 
frequency blueshift of this mode upon increasing temperature. The unusual hardening behavior of the lowest-frequency $E_{\mathrm{g}}$ mode was explained by Gao and others $[25,26]$ through three- and four-phonon processes where three-phonon anharmonic processes dominate over the four-phonon anharmonic interaction. If we have in mind that three-phonon decay (scattering) processes bring to mode softening while the quartic terms may be either positive or negative [8] we deduced that only quartic terms (see Eq. (3)) dominate and suppress the contribution from thermal expansion and from cubic anharmonic terms. This means that contrary to Gao and other authors temperature dependence of the main Raman mode seeks for new explanation and this will be strong motiv for our further investigation.

\section{Conclusion}

We examined the effects of temperature-induced changes on vibrational states of nanocrystalline materials like $\mathrm{CeO}_{2-\delta}$, ceria doped with $\mathrm{Nd}(\mathrm{Gd})$ and $\mathrm{TiO}_{2-\delta}$ using the Raman spectroscopy. At high temperatures, in pure and Nd doped ceria nanomaterials, four-phonon anharmonic processes dominate over three-phonon processes and the size effects have less influence on the Raman mode behavior than anharmonic effects. In a case of $\mathrm{Gd}$ doped ceria nanocrystals up to $300^{\circ} \mathrm{C}$ four-phonon coupling dominates but with further temperature increase the three-phonon coupling prevails indicating that the particles are grown enough to behave similar to polycrystalline sample. The Raman spectra after heat treatment demonstrated that these samples are still in nanometric range but anharmonic behavior is similar to their bulk counterparts. The unusual hardening of the lowest-frequency $E_{\mathrm{g}}$ mode in anatase nanopowders can be assigned to the intrinsic anharmonicity due to the dominant contribution of positive quartic terms.

\section{Acknowledgments}

This work was supported by the Ministry of Science and Technological Development of Republic of Serbia under the project No. 141047, the OPSA-026283 Project within the EC FP6 Programme and SASA project F-134.

\section{References}

[1] G. Morell, W. Pérez, E. Ching-Prado, R.S. Katiyar, Phys. Rev. B 53, 5388 (1996).

[2] N.S. Yuksek, N.M. Gasanly, A. Aydinli, J. Raman Spectrosc. 35, 55 (2004).

[3] P. Verma, S.C. Abbi, K.P. Jain, Phys. Rev. B 51, 16660 (1995).
[4] J. Serrano, M. Cardona, T.M. Ritter, B.A. Weinstein, A. Rubio, C.T. Lin, Phys. Rev. B 66, 245202 (2002).

[5] K.A. Alim, V.A. Fonoberov, A.A. Balandina, Appl. Phys. Lett. 86, 053103 (2005).

[6] M.X. Gu, L.K. Pan, B.K. Tay, C.Q. Sun, J. Raman Spectrosc. 38, 780 (2007).

[7] S. Boskovic, D. Djurovic, Z. Dohcevic-Mitrovic, Z. Popovic, M. Zinkevich, F. Aldinger, J. Power Sources 145, 237 (2005).

[8] A.A. Maradudin, A.E. Fein, Phys. Rev. 128, 2589 (1962).

[9] J. Kulda, A. Debernardi, M. Cardona, F. de Geuser, E.E. Haller, Phys. Rev. B 69, 045209 (2004).

[10] P.G. Klemens, Phys. Rev. 148, 845 (1966).

[11] T.R. Hart, R.L. Aggarwal, Benjamin Lax, Phys. Rev. B 1, 638 (1970).

[12] M. Balkanski, R.F. Wallis, E. Haro, Phys. Rev. B 28, 1928 (1983).

[13] Z.V. Popović, Z. Dohčević-Mitrović, A. Cros, A. Cantarero, J. Phys., Condens. Matter 19, 496209 (2007).

[14] I. Kosacki, V. Petrovsky, H.U. Anderson, P. Colomban, J. Am. Ceram. Soc. 85, 2646 (2002).

[15] Z.D. Dohčević-Mitrović, M.J. Šćepanović, M.U. Grujić-Brojčin, Z.V. Popović, S.B. Bošković, B.M. Matović, M.V. Zinkevich, F. Aldinger, Solid State Commun. 137, 387 (2006).

[16] M.D. Hernandez-Alonso, A.B. Hugria, A. Martinez-Arias, J.M. Coronado, J.C. Conesa, J. Soria, M. Fernandez-Garcia, Phys. Chem. Chem. Phys. 6, 3524 (2004).

[17] H. Richter, Z.P. Wang, L. Ley, Solid State Commun. 39, 625 (1981).

[18] I.H. Campbell, P.M. Fauchet, Solid State Commun. 58, 739 (1986).

[19] T. Sato, S. Tateyama, Phys. Rev. B 26, 2257 (1982).

[20] W.H. Weber, K.C. Hass, J.R. McBride, Phys. Rev. B 48, 178 (1993).

[21] Z.D. Dohčević-Mitrović, M. Radović, M. Šćepanović, M. Grujić-Brojčin, Z.V. Popović, B. Matović, S. Bošković, Appl. Phys. Lett. 91, 203118 (2007).

[22] S. Aškrabić, Z.D. Dohčević-Mitrović, M. Radović, M. Śćepanović, Z.V. Popović J. Raman Spectrosc., DOI 10.1002/jrs.2177.

[23] H. Hayashi, M. Kanoh, C.J. Quan, H. Inaba, S. Wang, M. Dokiya, H. Tagawa, Solid State Ionics 132, 227 (2000).

[24] K.R. Zhu, M.S. Zhang, Q. Chen, Z. Yin, Phys. Lett. A 340, 220 (2005).

[25] T. Ohsaka, J. Phys. Soc. Japan 48, 1661 (1980).

[26] K. Gao, Physica B 398, 33 (2007). 